\title{
High-Speed Infrared Imaging for Characterization of the Additive Manufacturing Process
}

\author{
by M.A. Langevin*, A. Huot*, S. Boubanga*, P. Lagueux*, E. Guyot* \\ *Telops, 100-2600 St-Jean-Baptiste ave., G2E 6J5, Québec, Québec, Canada, alexandrine.huot@telops.com
}

\begin{abstract}
Additive manufacturing is a method that allows rapid fabrication of metal pieces with various compositions, shapes and sizes. However, the extremely high thermal gradients involved in this fabrication process can induce stress and deformation in the materials. In an effort to better understand and visualize these thermal transfers, high-speed infrared imaging of the Direct Metal Laser Sintering (DMLS) process on stainless steel was carried out at 2800 frames per second with the Telops FAST M2k infrared camera. The results illustrate how high-speed infrared imaging can provide detailed information about thermal transfers in metal parts during such an industrial process.
\end{abstract}

\section{Introduction}

Additive manufacturing has gained popularity for the fabrication of metal parts over the last few years because of its great versatility. However, highly localized heating and cooling during the printing process can induce thermal expansion and contraction, resulting in residual stress and distortions that can affect the mechanical properties and dimensional stability of the metal parts. The thermal transfers are affected by many factors such as the material, speed and power of the heat source, geometry and thickness of the metal parts, the scanning strategy, powder thickness under the part, etc. [1] For example, due to edge effects, a heat source may interact with a region of the metal part before the heat from the previous pass had time to dissipate, resulting in more defects. Therefore, the design of the part and the printing strategy must be optimized according to the heat transfers during the manufacturing. The detection of such abnormalities at early stages of the printing process would save valuable time and material. Thermal post-processing can also be used to improve their mechanical properties, but the thermal history of the metal parts must be well known to be applied correctly.

Thermocouples are commonly used to measure the temperature of the substrate. However, they cannot measure temperature variations in the printed material. Pyrometers are widely used to measure such variations, but only infrared cameras allow a full layer thermal analysis because of their high spatial resolution. Furthermore, due to the highspeed of the lasers used in DMLS printers (up to $7.0 \mathrm{~m} / \mathrm{s}$ ), the acquisition speed must be considered for accurate characterization.

In this work, high-speed infrared imaging was carried out during the printing of stainless steel parts by DMLS. The results illustrate how high-speed imaging in the mid-wave infrared (MWIR, $3-5 \mu \mathrm{m}$ ) spectral range can bring some insights for material characterization during the additive manufacturing process.

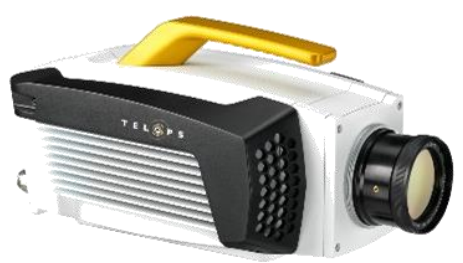

Fig. 1. Telops FAST M2k infrared camera.

\section{Experimental Information}

\subsection{Telops FAST M2k}

The Telops FAST M2k (see Figure 1) is a cooled high-performance infrared camera featuring a $320 \times 256$-pixel indium antimonide ( $\mathrm{InSb}$ ) focal plane array (FPA) detector covering the $3-5.5 \mu \mathrm{m}$ spectral range. A 50 -mm Janos lens was used for this experiment. A $256 \times 200$-pixel sub-portion of the FPA detector was used for imaging at $2800 \mathrm{fps}$. The camera is equipped with a 4-position internal filter wheel that allows the storing of commercially available 1 -inch (2.54 $\mathrm{cm}$ ) filters. The filter and calibration temperature range chosen for this experiment allowed us to monitor the thermal transfers occurring at temperatures much lower than the fusion temperature of the material. 


\subsection{D Metal Printing}

The metal parts were manufactured with the EOS-M290 Direct Metal Laser Sintering (DMLS) printer equipped with a $400 \mathrm{~W}$ Yb:Fiber laser. For this experiment, the printing of an umbrella stand was studied (Figure 2). The infrared images were acquired during the printing of the metal support, which consists of a tight metal grid and five full-metal anchors. For each monolayer of metal, the powder thickness was $40 \mu \mathrm{m}$. The power of the laser was set to $100 \mathrm{~W}$ for the grid and $290 \mathrm{~W}$ for the anchors. The speed of the laser was set to $675 \mathrm{~mm} / \mathrm{s}$. During the printing process, the platform was maintained at $150^{\circ} \mathrm{C}$. Since the additive manufacturing process is extremely sensitive to oxygen, the printing must be carried out under inert atmosphere. However, the silica window typically used in the observation port is opaque to mid-infrared wavelengths. In this experiment, a $2 \mathrm{~mm}$ thick sapphire window was used instead. Imaging is achieved by looking at a $50.8 \mathrm{~mm}$ gold mirror fixated above the observation port with a $45^{\circ}$ angle (see Figure 2). The material used for the manufacturing of this part was $316 \mathrm{~L}$ stainless steel (Fe, $<0.03 \% \mathrm{C}, 16-18.5 \% \mathrm{Cr}, 10-14 \% \mathrm{Ni}, 2-3 \% \mathrm{Mo},<2 \% \mathrm{Mn}$, $<1 \% \mathrm{Si},<0.045 \% \mathrm{P},<0.03 \% \mathrm{~S})$.

\section{Results and Discussion}

The images recorded by the camera are in radiometric temperature, which consider an emissivity $(\varepsilon)$ of 1 . Therefore, to measure a thermodynamic temperature, $\varepsilon$ must be known. Depending on the surface condition, the emissivity of stainless steel is significantly different. While weathered stainless steel has a $\varepsilon$ of 0.85 , the polished metal has a $\varepsilon$ of 0.075 . [2] Considering that the printed metal was slightly shiny, $\varepsilon$ was approximated to 0.4 , which is consistent with the value used by other research groups in similar conditions. [3]
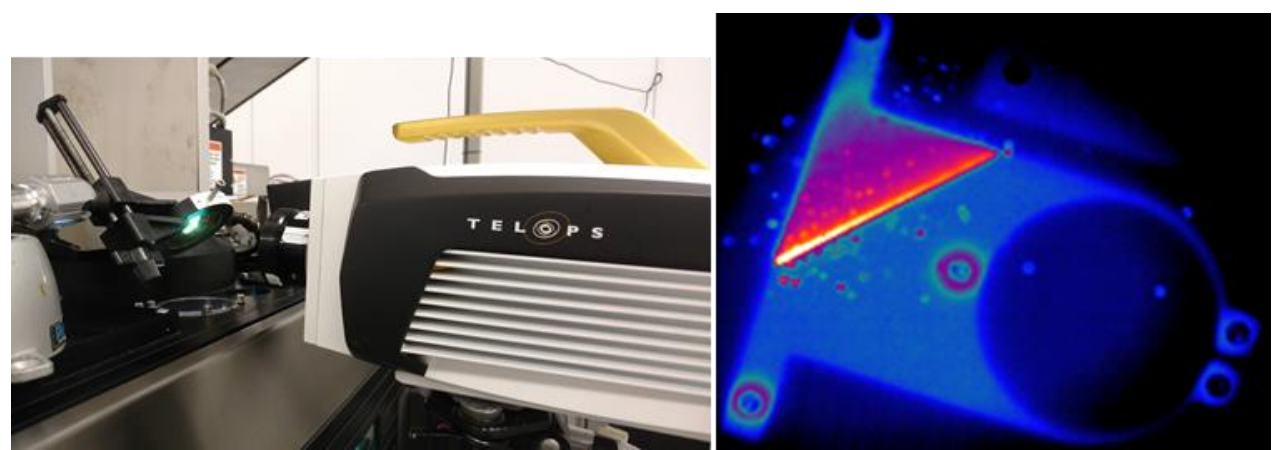

Fig. 2. Experimental setup for infrared imaging of the additive manufacturing process and a typical image obtained with the FAST M2k.

\subsection{Printing of the umbrella stand}

The printing process of the support can be separated into three distinct steps (Figure 3 B-E). First, five full-metal spots are printed. These spots are solid anchors fixing the piece to the base platform. Then, the laser sweeps the surface of the metal piece to make a tight grid. Finally, the contour of the piece is sintered by the laser to improve the surface finish. The thermal profile as a function of time was traced for the five points presented in Figure 3(A):

- $\quad$ Point $A$ - On a full-metal anchor in the middle of the metal piece.

- Point B - On a narrow part of the piece, between a full-metal anchor and a corner.

- Point C - At the center of the metal grid, far from the edges and the anchors.

- Point D - On the narrow circular section of the of the umbrella stand.

- Point E - On the narrow circular section of the umbrella stand, near a full-metal anchor. 
(A)

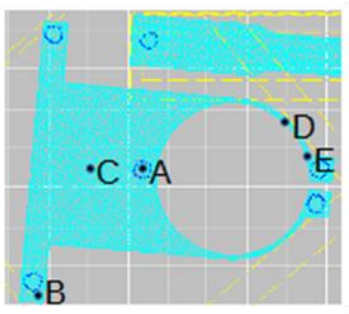

Fig. 3. (A) Layout used
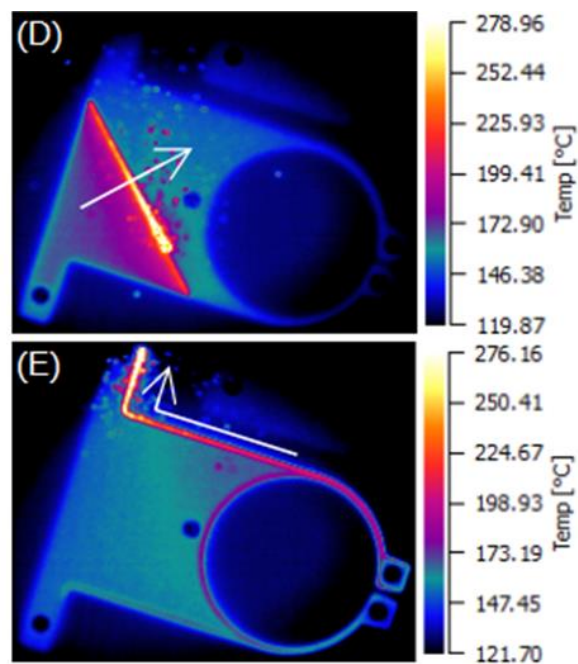

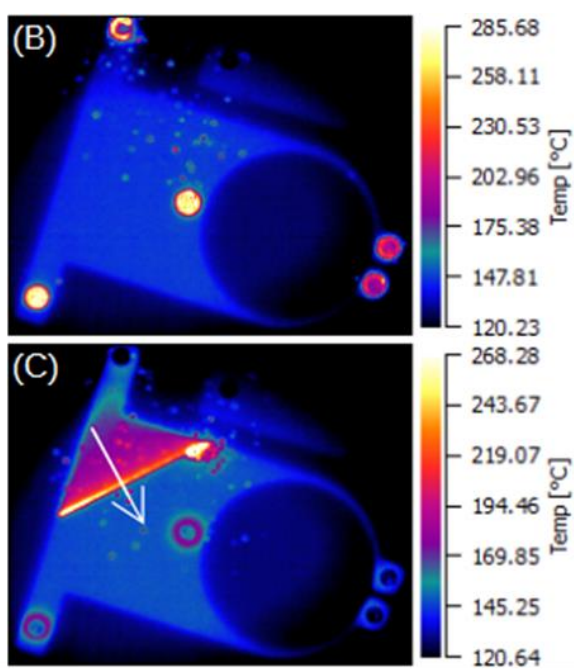

Printing of the grid, (E) Sintering of the edges.

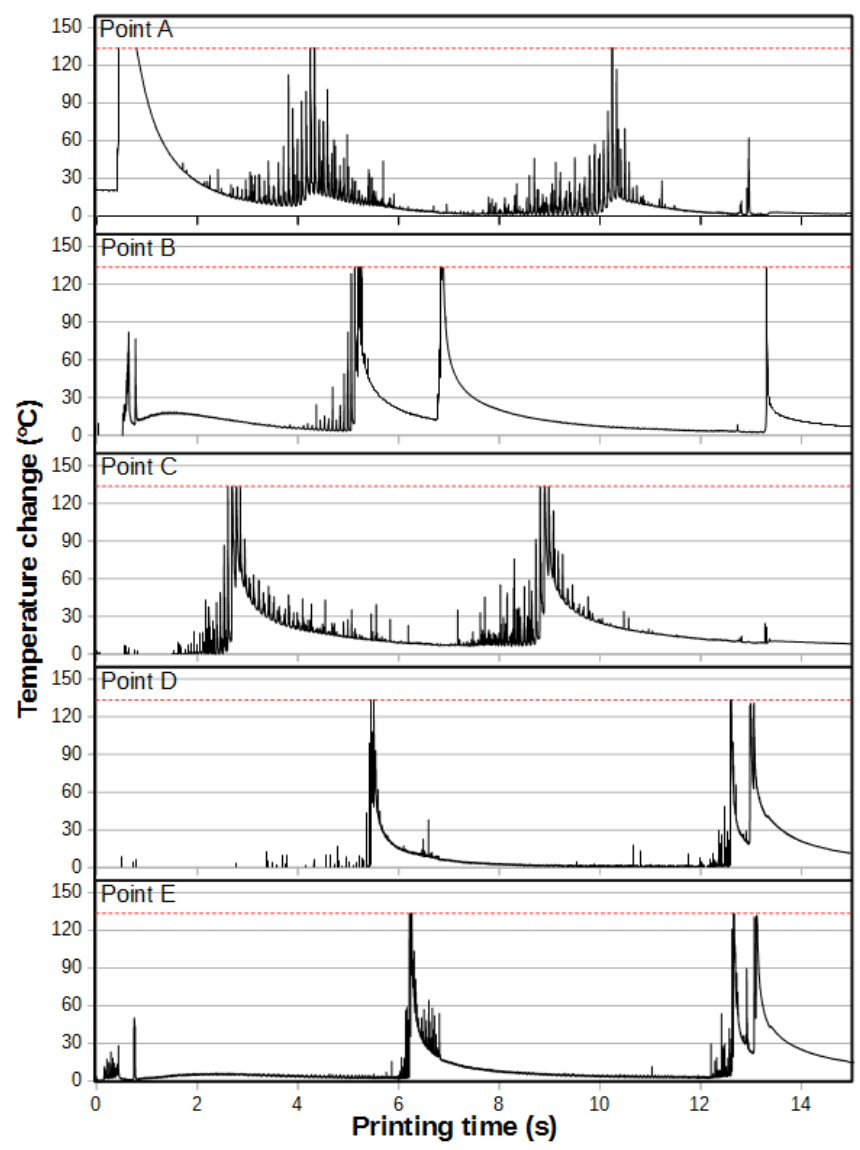

Fig. 4. Temperature change as a function of the printing time for points $A$ to $E$.

The thermal profiles for each point are presented in Figure 4. Depending on the position on the metal piece, the results obtained were significantly different. These thermal profiles can easily be correlated with the events presented in Figure 3. For point $A$, which is on a full-metal anchor, we first observed a rapid increase of temperature. Due to the high temperature of molten metal, saturation is observed for $350 \mathrm{~ms}$. The temperature then exponentially decreases for the next three seconds. Between 2.7 and 6.2 seconds, temperature spikes are also observed every 60 to $80 \mathrm{~ms}$, due to the laser printing the grid. A similar pattern is observed for the second passage of the laser. 
Point $B$ is located between a full-metal anchor and the edge of the piece. We first observed a temperature increase of $86^{\circ} \mathrm{C}$ during the printing of the anchor. The quick cooling is followed by a slight temperature increase. This is most likely caused by diffusion of heat from the anchor. This phenomenon can be well seen on many infrared images such as Figure 2 and Figure 3(C). A similar trend was observed at point E. We then observed an increase of temperature up to saturation because of the two passages of the laser printing the grid. Since this part of the metal piece is close to a corner, the heat spikes from the grid printing are not as important as they are for point $A$ and $C$. Finally, at 13.3 seconds, the detector is briefly saturated, during the passage of the laser sintering the edges of the metal piece.

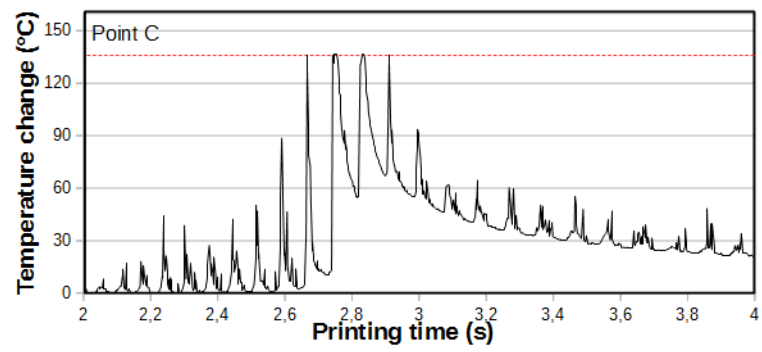

Fig. 5. Thermal profile for points $C$ zoomed between 2 and 4 seconds.

Point $\mathrm{C}$ is located in the middle of the grid. Therefore, this point is highly affected by numerous passages of the laser in the surrounding area. A zoom between 2 and 4 seconds, is presented in Figure 5 . Progressively increasing temperature spikes can be observed every 60 to $80 \mathrm{~ms}$, until 2.92 seconds due to the approaching laser. Those spikes then become progressively less intense as the laser moves away. Irregular temperature spikes can also be seen during the process. They might be caused by hot particles that are ejected at the passage of the laser. These hot particles can be easily seen in Figure 2 and Figure 3 . Since point $C$ is far from the edges, the temperature was not affected during the surface finishing.

Points $D$ and $E$ are located on the thin circular part of the metal piece. Those two thermal patterns are similar, with temperature increases from the grid printing in both directions and the sintering of the edges. For point $\mathrm{E}$, the slow heat increase of about $10^{\circ} \mathrm{C}$ due to the nearby full-metal anchor was also observed between 1 and 4 seconds. Since the edges are sintered in the inner and outer parts of the circle, temperature increases from the two passages could be observed. Because of the high speed of the laser $(675 \mathrm{~mm} / \mathrm{s})$, this section of the pattern can only be compared by zooming between 12.5 and 13.5 seconds (Figure 6 ).

First, we see a temperature increase from the second passage of the laser printing the grid at 12.60 and 12.58 seconds for point $D$ and $E$ respectively. The temperature increases from the laser sintering the edges is observed at 13.01 and 13.08 seconds for point $D$ and at 13.03 and 13.07 seconds for point $E$. Since, those two laser passages are only separated by 70 and $40 \mathrm{~ms}$ respectively, the high speed of the camera is essential for an accurate comparison of those two patterns.

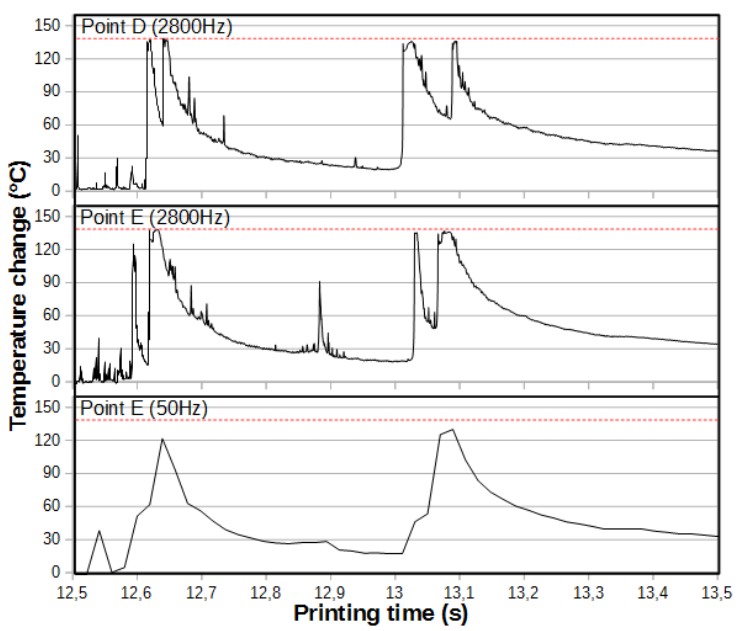

Fig. 6. Thermal profile for points $D$ and E zoomed between 12.5 and 13.5 seconds. 
Figure 6 presents the difference between results acquired for point $\mathrm{E}$ at $2800 \mathrm{~Hz}$ and $50 \mathrm{~Hz}$, which corresponds to the speed of most microbolometers. Here, $1 / 56$ points of the thermal profile acquired at $2800 \mathrm{~Hz}$ were used to simulate an acquisition speed of $50 \mathrm{~Hz}$. This profile does not allow to observe the separation between the temperature increase from the sintering of the inner and outer part of the metal part. The exponential temperature decrease is also not as well defined as it is at $2800 \mathrm{~Hz}$. Furthermore, for this experiment, the laser speed was much slower than the maximum speed of $7.0 \mathrm{~m} / \mathrm{s}$ that can be achieved with DMLS 3D printers. This highlights the importance of using an high-speed camera for accurate material characterization in additive manufacturing applications.

\subsection{Visualization of the melt pool}

Until now, the results focussed on relatively low temperature heat transfers in the metal part. Using a neutral density filter, it is also possible to visualize the melt pool at very high temperatures during the process. Since lack of fusion or overheating can affect the porosity of the manufactured material, it is also an important parameter to monitor. Figure 7 shows the melt pool for a section of the umbrella stand. Here, the laser power was set to $290 \mathrm{~W}$ and the speed to $1115 \mathrm{~mm} / \mathrm{s}$. More results about the melt pool characterization using Telops high-speed infrared cameras will be presented in future works.

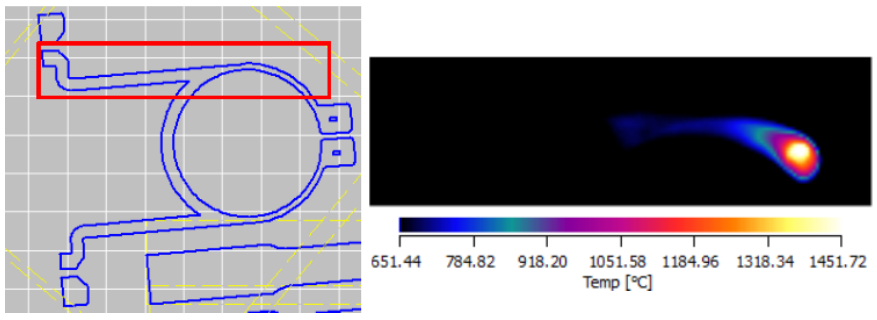

Fig. 7. Visualization of the melt pool on a section of the umbrella stand.

\section{Conclusion}

The thermal profiles on different points of a metal part printed by additive manufacturing were successfully traced to monitor the fabrication process. The use of neutral density filters for visualization of the melt pool was also presented. This type of results can be useful for improving the design of the metal parts and optimisation of the printing strategy in order to minimize the creation of residual constraints. The results presented in this application note demonstrate how high-speed infrared imaging combined with good spatial resolution is a valuable tool for characterisation of a great variety of thermal patterns, even in the same piece.

\section{Acknowledgments}

Telops would like to acknowledge the support of Olivier Marcotte and Martin Dallaire from the Centre de recherche industrielle du Québec (CRIQ) in this work.

\section{REFERENCES}

[1] Sames, W. J., et al., The Metallurgy and Processing Science of Additive Manufacturing, Int. Mater. Rev. (2016).

[2] The Engineering Toolbox. (2017). http://www.engineeringtoolbox.com/emissivity-coefficients-d_447.html

[3] Lane, B., et al., Thermographic Measurements of the Commercial Laser Powder Bed Fusion Process at NIST, Rapid Prototyp J. (2016).

[4] Liu, H., et al., Residual Stress and Deformation Modelling for Metal Additive Manufacturing Processes, MCM Conference (2015), Barcelona, Spain, Paper No. 245. 